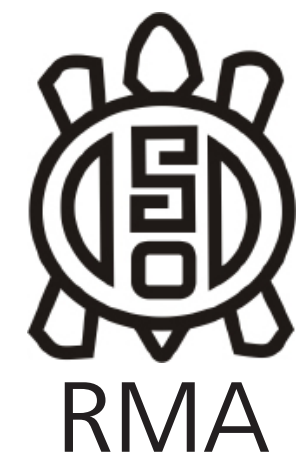

Antropología Social

\title{
La inversión de la teoría en la etnografía en antropología social
}

\author{
The investment of theory in ethnography in social anthropology
}

Fernando Alberto Balbi*

*Universidad de Buenos Aires. Facultad de Filosofía y Letras. Instituto de Ciencias Antropológicas. Sección Antropología Social. CONICET. Buenos Aires, Argentina. E-mail: fabalbi@yahoo.com.ar

\begin{abstract}
Resumen
En este artículo analizo la forma en que opera la teoría en la investigación etnográfica en la antropología social y/o cultural. Argumento que la etnografía supone el despliegue de ciertos procedimientos analíticos que conducen a relativizar a la teoría - y, eventualmente, a reformularla - a partir de su confrontación con los materiales etnográficos: la integración dinámica de las perspectivas nativas en el análisis; la combinación del holismo, la restricción de la preselección de los materiales etnográficos y su estricta contextualización; y diversos usos de la comparación. A continuación, ilustro esta afirmación mostrando cómo mis etnografías de los conflictos entre los asociados de una cooperativa de pescadores y del concepto peronista de 'lealtad' dieron lugar a algunos desarrollos teóricos en torno del análisis de la dimensión moral de la vida social. Finalmente, sugiero que esos procedimientos son el correlato al nivel de la metodología de la 'perspectiva etnográfica', una teoría muy general sobre la naturaleza de la vida social que ha sido desarrollada a lo largo de la historia de la disciplina.
\end{abstract}

Palabras clave: Antropología social; Etnografía; Teoría; Metodología; Perspectiva etnográfica.

\begin{abstract}
In this article I analyze how theory operates in ethnographic research in social and/or cultural anthropology. I argue that ethnography involves the deployment of certain analytical procedures that lead to the relativization of theory - and, eventually, to its reformulation - through its confrontation with ethnographic materials: the dynamic integration of the native perspectives in the analysis; the combination of holism, the restriction of the preselection of ethnographic materials, and their strict contextualization; and various uses of comparison. Next, I illustrate this statement by showing how my ethnographies of the conflicts between the members of a fishermen's cooperative and of the Peronist concept of 'loyalty' gave rise to some theoretical developments around the analysis of the moral dimension of social life. Finally, I suggest that these procedures are the correlate at the level of methodology of the 'ethnographic perspective' - a very general theory about the nature of social life that has been developed throughout the history of the discipline.
\end{abstract}

Keywords: Social anthropology; Ethnography; Theory; Methodology; Ethnographic perspective.

En contraste con lo que sucede en otras disciplinas, donde suele ser considerada como un método entre otros posibles, es común entre los antropólogos el dar por sentado que la etnografía es mucho más que un método: se trataría, en cambio, de una práctica de investigación distintiva de nuestra disciplina, el núcleo de su productividad específica, una suerte de experiencia profundamente transformadora que habilitaría a los antropólogos que la practican a producir un tipo de conocimiento particular y, desde luego, superior a todos los producidos por colegas de otras especialidades (Marcus, 2016; Howell, 2017, 2018; Shah, 2017). Desgraciadamente, y precisamente porque se invierte tanto trabajo en sostener esta idea, predomina en la antropología social y/o cultural ${ }^{1}$ una tendencia dar por sentado respecto de la etnografía ${ }^{2}$ más de lo que se analiza y, hasta cierto punto, a mistificarla.

\footnotetext{
${ }^{1}$ A fin de simplificar la exposición, en adelante escribiré simplemente 'antropología'. Es menester aclarar que me ocuparé exclusivamente de la antropología en cuanto disciplina académica y no abordaré las perspectivas que tienden disolver las barreras entre las antropologías 'académicas' y 'no académicas' (Escobar y Restrepo, 2005). Por lo demás, cabe advertir que me considero un antropólogo social y no 'cultural' o 'sociocultural', y que escribo en tanto tal; véase: Balbi (2015).

2 En la medida de lo posible, y a fin de evitar repeticiones incómodas, en adelante escribiré 'etnografía' sin aclarar que me refiero a su práctica en el campo de la antropología.
} 
Sería imposible recorrer extensamente la literatura en estas páginas para, ya que no probarla (pues, evidentemente, la acumulación inductiva de ejemplos nunca es probatoria), al menos delinear los alcances exactos de esta generalización que pronuncio y que, como toda generalización, desatiende en alguna medida a las variaciones que se presentan en la realidad del mundillo antropológico. ${ }^{3}$ Un índice bastante claro de esta tendencia es la frecuencia con que los antropólogos reaccionan escandalizados cada vez que algún colega osa poner en duda el valor axiomático de la etnografía. Por ejemplo, en un episodio reciente, la pretensión de Tim Ingold $(2017)^{4}$ en el sentido de que la puesta en valor de la antropología exige separarla radicalmente de la etnografía ha dado lugar a una serie de enfáticas reafirmaciones de la importancia de ésta que se extienden entre las relativamente meditadas (MacDougall, 2016; Marcus, 2016; Cook, 2016; Shryock, 2016; Shah, 2017) y las que se reducen, en última instancia, a la repetición más o menos disimulada de lugares comunes (Da Col, 2017; Howell, 2017; Miller, 2017). Acaso también valga la pena apuntar que, incluso en medios periféricos como lo es el de la antropología argentina, es común escuchar, tanto en eventos públicos (conferencias, paneles, presentaciones en congresos, presentaciones de libros, etc.) como en conversaciones entre colegas, que la etnografía es lo que nos distingue de los colegas de disciplinas limítrofes y que nos habilita a producir conocimientos que escapan a sus posibilidades.

Otro índice importante de la tendencia a mistificar la

\footnotetext{
${ }^{3}$ Hace ya más de veinte años, Akhil Gupta y James Ferguson (1997) se quejaban de que, a pesar de la etnografía estaba siendo practicada de maneras cada vez más deslocalizadas, "demasiado frecuentemente, el marco disciplinario institucionalizado de recepción y evaluación sigue viendo al conocimiento experiencial 'basado en el campo' como el núcleo privilegiado" (p. 37; la traducción de esta y otras citas de originales en lenguas extranjeras me pertenece) del trabajo etnográfico. En mi opinión, la tendencia que favorecían estos autores era y sigue siendo secundaria respecto de las miradas hegemónicas en las academias centrales. Hoy en día, si bien raramente se pone en duda la importancia de la 'multilocalidad' y del uso de documentos -las prácticas por entonces crecientes a que se refieren los autorescomo aspectos centrales del quehacer etnográfico, los autores afiliados a las instituciones académicas centrales y que publican con mayor frecuencia en las revistas más reputadas siguen por lo general asociando la distintividad y la relevancia de la etnografía a su componente experiencial basado en el campo.

${ }^{4}$ El artículo de Ingold fue publicado originalmente en 2014 con el título "That's enough about ethnography!" en el número 4(1) de Hau: Journal of Ethnographic Theory y se convirtió en el más descargado desde la web de la revista. Esto dio lugar tanto a una entrevista a Ingold y varias notas que fueron publicadas durante 2016 en un blog de la revista Cultural Anthropology, así como a un simposio desarrollado en ese mismo año en el Centre for Ethnographic Theory (SOAS, London) con el título "Dos o tres cosas que amo u odio acerca de la etnografía", cuyas ponencias (más algunos textos escritos a invitación de los editores) fueron luego publicados en $\mathrm{Hau}$, ya en 2017. Todo este movimiento en torno del artículo de Ingold muestra no sólo que su posición es disruptiva en el contexto de las academias hegemónicas sino, especialmente, que la incomodidad que genera - bien conocida al menos desde su conferencia en honor de Radcliffe-Brown (Ingold, 2008) - se hizo más acuciante cuando su texto de 2014 atrajo la atención de muchos lectores.
}

etnografía atañe directamente al punto que me interesa abordar en esta ocasión. Me refiero a un espectro de actitudes interrelacionadas que han llegado a predominar en las academias de la disciplina en los países centrales y a reflejarse ampliamente en las nuestras de la mano de la hegemonía de perspectivas interpretativistas y particularistas (Balbi, 2015, 2017a), todas las cuales tienen en común, como bien ha observado Roy Ellen (2010), el rebajar en mayor o menor medida la relevancia de la teoría en la investigación etnográfica En un extremo encontramos el abierto rechazo de la teoría concebida como una suerte de 'peligro' para la investigación antropológica (véase, por ejemplo: Yengoyan, 2006). En el otro, la minimización del papel de la teoría que se produce cada vez que se admite que toda etnografía tiene un fundamento teórico pero se insiste en afirmar que su productividad proviene del 'campo' mismo, de la experiencia sui generis que atraviesan los etnógrafos o, sencillamente, del carácter 'inductivo' de la etnografía (véanse, por ejemplo: Guber, 2016; Restrepo, 2016, Miller, 2017; Howell, 2017, 2018). Entre ambos extremos, finalmente, es habitual encontrarse con la celebración de la 'creatividad' o 'imaginación' del etnógrafo como fuentes últimas y fundamentales de la teoría (Da Col y Graeber, 2011; Da Col, 2017), de obscuros procedimientos dirigidos a 'bifurcar' o desestructurar su pensamiento para dar lugar a desarrollos originales (Strathern, 2004), o de las apropiaciones idiosincráticas de textos y autores particulares del campo de la filosofía desarrolladas por cada antropólogo acorde a sus preferencias individuales (Das, Jackson, Kleinman y Singh, 2014). Si se entiende por 'teoría' a un entramado conceptual internamente coherente, susceptible de ser operacionalizado para formular y abordar problemas relativos a la realidad, y desarrollado a partir de los esfuerzos dedicados a resolver otros problemas del mismo orden que han sido planteados previamente (Hastrup, 1997; Holy, 1984), resulta bastante claro que todas las actitudes que acabo de enumerar son más o menos antiteóricas. ${ }^{5}$

Continuando con una serie de reflexiones sobre la naturaleza de la investigación antropológica (Balbi, 2012, 2015, 2017a, 2017b), me propongo ir más allá de estas miradas mistificadas y antiteóricas sobre la etnografía para abordar el problema de cómo opera la teoría en su transcurso. Desde luego, la cuestión es demasiado compleja para abordarla aquí en toda su extensión. Por ello, me concentraré en el análisis de un asunto más estrecho y manejable: la forma en que las orientaciones teóricas específicas de cada antropólogo —es decir, aquellas que él o ella despliega para construir su objeto de investigación y definir los problemas a abordar (Holy, 1984) - se relacionan con sus materiales en el curso de una investigación etnográfica. A este respecto, mostraré

\footnotetext{
${ }^{5}$ La amplia difusión de prejuicios antiteóricos y la mistificación de la etnografía parecen ser dos de las condiciones centrales de la 'crisis' disciplinaria sobre la que diversos autores han advertido a lo largo de las últimas décadas (Bloch, 2005; Ingold, 2008; Da Col y Graeber, 2011; Visacovsky, 2017).
} 
que la etnografía supone el despliegue de ciertos procedimientos analíticos que conducen a relativizar a la teoría y, eventualmente, a reformularla a partir de su confrontación con los materiales. Asimismo, recapitularé un par de momentos de mis propias investigaciones etnográficas a fin de esclarecer mi argumento. Finalmente, me referiré muy brevemente al problema, mucho más complejo, de los fundamentos teóricos de la totalidad de la empresa etnográfica, que residen en una teoría muy general sobre la naturaleza de la vida social que se ha desarrollado a lo largo de la historia de la antropología y cuyos correlatos metodológicos son, precisamente, esas orientaciones analíticas que inducen a los etnógrafos a relativizar sus orientaciones teóricas específicas.

\section{La inversión de la teoría en los hechos}

Como he adelantado, me interesa examinar el modo en que operan en la investigación etnográfica las orientaciones teóricas específicas a que apela cada antropólogo, es decir, las que cada uno de nosotros despliega para construir su objeto de investigación y definir los problemas a abordar (Holy, 1984). Podría pensarse que lo adecuado en este contexto es hacer referencia a 'una' teoría pero, en mi opinión, lo más común es que los antropólogos no cuenten con una teoría en sentido estricto sino con uno u otro conjunto de orientaciones teóricas específicas (que además, como veremos luego, son cambiantes) provenientes de diversas fuentes y más o menos reformuladas en función de su mutua articulación. Aunque, en la práctica, pueden encontrarse tanto casos de adhesión estricta a una teoría como de una marcada falta de coherencia teórica, el primer caso es más o menos asimilable a la situación más frecuente mientras que lo propio del segundo es la caída por debajo del umbral del trabajo científico/académico estándar y carece de interés a mis fines.

Aunque resulte relativamente sorprendente en una disciplina que lleva cerca de cuarenta años haciendo de la crítica de sus propios fundamentos una práctica casi obsesiva, muchos antropólogos siguen creyendo que la teoría deriva de la observación (Ellen, 2010) y que la etnografía es una modalidad de investigación inductiva (Miller, 2017; Howell, 2018). Sin embargo, la realidad no puede ser abordada sino desde uno u otro punto de vista teórico (Bachelard, 1979; Bourdieu, Chamboredon y Passeron, 1986; Putnam, 1981): en efecto, el centro de gravedad de toda investigación científica es, necesariamente, la teoría en la medida en que "lo real no tiene nunca la iniciativa puesto que sólo puede responder si se lo interroga" (Bourdieu et al., 1986, p. 55). Según la entiendo, entonces, la investigación antropológica como cualquier otra forma de investigación científicaconstruye un objeto de investigación en función de una problemática teórica y, a lo largo de todo su desarrollo, despliega recursos heurísticos que ponen a la teoría en acción para producir afirmaciones sobre hechos particulares y generalizaciones que son apenas juicios de valor de carácter teórico y estrictamente provisionales (Balbi, 2015, 2017a). Por ende, no puede decirse que las teorías proceden de los hechos: al contrario, el despliegue de la teoría da lugar a la producción teórica a través de un camino representado por los hechos (Balbi, 2015). Así, pues, lo que me interesa en este apartado es explorar la forma que asume en la etnografía ese camino de las orientaciones teóricas específicas del antropólogo a través de los hechos.

Existen, sin duda, muchas formas diferentes de abordar esta cuestión pero no creo haber leído ninguna mejor que la que se nos ofrece en el prefacio escrito en 1976 por Moacir Palmeira (2011) para la primera edición de $E I$ vapor del Diablo, de José Sergio Leite Lopes. Según el autor, en la etnografía de Leite Lopes la teoría es 'invertida en los hechos.' Con esto, Palmeira (2011) quiere decir que el análisis se nutre de problemas teóricos que no son abstractos sino "que se imponen a partir de análisis de una situación concreta" (p. 57). Esto, afirma, tiene consecuencias tanto para la teoría como para los datos. Al ser puesta en acción, la teoría deja de ocupar un lugar idealizado en cuanto una "meta-teoría intocada e intocable" (Palmeira, 2011: 58) para, en cambio, pasar a significar "una colocación en una relación orientada de 'hechos' o 'datos' que de otro modo no tendrían sentido" (Palmeira, 2011, p. 58). De esta forma, afirma, la teoría necesariamente "va más allá de los 'hechos', volviéndose un instrumento para el conocimiento de otros 'hechos', aunque no puede remplazar al análisis de esos otros 'hechos' de ninguna manera" (Palmeira, 2011, p. 58).

El proceder examinado por Palmeira da cuenta simultáneamente de la productividad de la etnografía en los planos de la teoría y del análisis de los casos abordados. Por un lado, puesto que se la operacionaliza en función de situaciones particulares, "la teoría supone necesariamente un 'trabajo teórico'" (Palmeira, 2011, p. 58), es decir, la producción de teoría en el curso mismo de la etnografía. Por el otro, e incluso por definición, los datos "son construidos" (Palmeira, 2011, p. 59): en efecto, se los construye apelando a los materiales producidos en el campo pero en función de la teoría, en el sentido de que "sólo existen a partir de preguntas" (Palmeira, 2011, p. 59). Así, podríamos decir, se hace posible producir, en un mismo movimiento analítico, resultados etnográficos y teóricos: pues si, por un lado, el análisis del caso - la etnografía - se activa y ordena en relación con la teoría 'invertida' a tal efecto, ocurre por el otro que esa inversión da frutos que "no significan el rechazo o la confirmación de una teoría; significan la relativización in actu de conjuntos de proposiciones" $y$, así, "la construcción de nuevas variantes y la apertura nuevos caminos, sin los cuales la teoría no puede vivir" (Palmeira, 2011, p. 59).

¿Pero cómo, concretamente, se 'invierte' la teoría en una 
investigación etnográfica? Responder este interrogante exige examinar brevemente algunos procedimientos característicos de la etnografía que, precisamente, conducen a construir los datos de maneras tales que ponen en tensión a las orientaciones teóricas y hacen de la relativización de la teoría su forma de 'vida' propiamente etnográfica.

\section{La integración dinámica de las perspectivas nativas en la etnografía}

El primer procedimiento característico de la etnografía es lo que en otro lugar he denominado "la 'integración dinámica' o 'analítica' de las perspectivas nativas en la descripción etnográfica" (Balbi, 2012, p. 493). ${ }^{6}$ Las perspectivas nativas son universos de referencia compartidos por ciertos sujetos socialmente situados, articulaciones cambiantes de prácticas y sentidos sólo en parte verbalizables que organizan su actividad y resultan en parte de esta y en parte de la de otros sujetos. Se trata, desde luego, de construcciones heurísticas desarrolladas por los etnógrafos, y no de la mera transcripción del entendimiento que los sujetos tienen efectivamente respecto de su mundo social (Balbi 2012): en este sentido, Rosana Guber (1991) afirma que lo que ella denomina "perspectiva del actor (...) tiene existencia empírica aunque su formulación, construcción e implicancias estén definidas desde la teoría" (p. 75). ${ }^{7}$ Ese substrato empírico tiene una importancia fundamental porque se trata de la única base razonable para sostener uno de los supuestos básicos de la etnografía de cuño clásico: el de que las perspectivas nativas constituyen un camino privilegiado para acceder al conocimiento de lo social no sólo porque son parte de ello sino, particularmente, porque los actores deben tener algún tipo de visión de su propio mundo social tal que les permita operar en él, lo que no significa que sean 'objetivamente' válidas o adecuadas en términos empíricos pero sí, necesariamente, que han de guardar alguna correspondencia - a priori indeterminada en su tipo y en su grado - con ese mundo tal que permita habitarlo (Balbi, 2012).

Al hablar de la integración dinámica de las perspectivas nativas en la investigación etnográfica me refiero a su incorporación paulatina, siempre incompleta, orientada

\footnotetext{
6 He desarrollado esta caracterización de la forma en que las perspectivas nativas son puestas en juego en la etnografía como un intento de superar algunos problemas inherentes a la metáfora del 'diálogo' entre las 'teorías' nativas y del etnógrafo que presenta Mariza Peirano (2002). Véase: Balbi (2012).

${ }^{7}$ Siguiendo una tradición consagrada por Bronislaw Malinowski, la construcción de la 'perspectiva nativa' o 'del actor' por los antropólogos tiende a fundarse en la totalidad del comportamiento observado (incluyendo las declaraciones de los actores pero también desbordándolas) y, más ampliamente, en el análisis de la materialidad del mundo social (organización del espacio, vestimenta, tecnología, etc.) en cuanto se encuentra cargada de sentido por la actividad humana. Mi uso del plural obedece a que las situaciones en que los sujetos relevantes para un análisis social comparten de manera homogénea un único universo de referencia son excepcionales. Véase: Balbi (2012)
}

a hacer de ellas piezas consistentes de la descripción analítica de una porción del mundo social. Se trata de una reiterada confrontación (producida a lo largo de todo el período de trabajo de campo y prolongada hasta el momento mismo de la redacción de los resultados finales) entre las perspectivas nativas y la perspectiva teóricamente informada del antropólogo que está orientada a sacar partido de la correspondencia relativa que existe entre aquellas y el mundo social que habitan los actores (Balbi, 2012; Guber, 1991). El procedimiento consiste en

“(...) colocar reiteradamente en tensión esos diferentes puntos de vista, asumiendo siempre que el nuestro no será adecuado hasta tanto no llegue a ser capaz de aprehender plenamente a las perspectivas nativas tornándolas, así, en partes integrales del análisis etnográfico, en piezas necesarias de la descripción analítica que, a la vez, resulten inteligibles por virtud de su integración en dicho contexto." (Balbi 2012, p. 492)

Así, las perspectivas nativas son 'integradas' en la descripción etnográfica en un doble sentido: primero, los intentos sistemáticos de producir esa integración son el motor fundamental del desarrollo de la etnografía, la pulsión que la mantiene en movimiento; y segundo, el análisis final (el texto etnográfico u otros tipos de escritos que incorporen descripciones etnográficas) debe dar cuenta de sus lógicas, fundamentos y vinculaciones con los procesos sociales examinados.

\section{Holismo, restricción de la preselección de los materiales y contextualización}

En segundo lugar, la etnografía se caracteriza por la apelación a tres procedimientos analíticos estrechamente interrelacionados que encuentran sus raíces en el trabajo de los fundadores de la antropología moderna y que se combinan para poner en tela de juicio la mirada del etnógrafo y forzarlo a revisar sus análisis parciales y las preferencias teóricas en que se basan. Primero, el holismo, entendido — de una manera contemporáneacomo una predisposición a tomar en consideración un amplio espectro de hechos, tratando de manera conjunta cuestiones que el saber académico burgués tiende a separar a priori como lo son la política, la economía, el parentesco y otras modalidades de relaciones personales, la ciencia, la religión, etc. (Shah, 2017; Hastrup, 2005; Guber, 1991). Se trata de una "visión totalizadora que hace posible un análisis abarcador de las formas sociales, las acciones individuales, las creencias colectivas, las restricciones materiales y las expresiones creativas" (Hastrup, 2005, p. 133). Segundo, la preferencia por evitar —en la medida de lo posible - la preselección de la información a relevar, que se refleja en la a veces abrumadora extensión de las notas de campo de los antropólogos y en el registro, especialmente durante 
las primeras etapas de una investigación, de gran cantidad de 'detalles' que parecen insignificantes pero que se hacen constar porque se asume que la cuestión de si son o no relevantes sólo podrá ser resuelta más adelante, en función de los avances del análisis detallado de la totalidad de los materiales (Sanjek, 2002; Guber, 1991). Y tercero, el imperativo de relevar la información, determinar su relevancia relativa y analizarla -en un primer y fundamental nivel de aproximación - en su contexto de origen, tratándola siempre como la expresión de un entramado de relaciones socialmente situado (Hastrup, 2005; Guber, 1991; Pitt-Rivers, 1973): el dato etnográfico sólo es tal en tanto y en cuanto se trata de este modo a cada pieza de información, resistiendo la tentación de arrancarla de su contexto originario como, al contrario, exigen hacerlo otros procedimientos cuyo uso es central en disciplinas limítrofes.

Estos tres procedimientos analíticos se suponen mutuamente: un análisis holístico exige limitar al mínimo posible la preselección de los materiales y examinar inicialmente la información en su contexto 'de origen' para poder determinar las líneas de relación que requieren ser examinadas; la reconstrucción analítica del contexto de cualquier asunto demanda acceder a toda la información que se revele eventualmente como potencialmente útil $y$, por ende, no segmentar los hechos por adelantado; el relevamiento de información extenso y poco dirigido sólo es factible si se evita segmentar la realidad antes de emprenderlo y, a la vez, cada pieza de información sólo es inteligible en la medida en que se la refiere a otras relevadas en el mismo contexto. Así, sus efectos, sean independientes o combinados, fuerzan al etnógrafo a revisar sus esquemas analíticos y orientaciones teóricas a la luz de un material siempre en expansión, típicamente impredecible y capaz de plantear relaciones que no ha contemplado a priori pero que debe ser capaz de integrar coherentemente a su descripción analítica.

\section{Usos de la comparación}

Finalmente, la etnografía habitualmente supone apelar a una variedad de formas de comparación, cosa que, lamentablemente, muchas veces sucede en la antropología actual sin demasiada autoconciencia y, en consecuencia, sin el debido control de los procedimientos empleados, sus alcances y limitaciones (Balbi, 2015, 2017a; Fox y Gingrich, 2002). Interesan aquí dos de los muchos aspectos que involucra el uso de la comparación en el marco de los análisis etnográficos.

Por un lado, como observa Roger Sanjek (2002), en cada paso de su labor, los antropólogos refieren los asuntos que analizan a un amplio espectro de condiciones sociales y culturales con las que están familiarizados a través de la literatura etnográfica, examinando las similitudes y diferencias entre sus materiales y los casos sobre los que han leído. De esta forma, evitan caer (o, por lo menos, lo intentan) en el natural etnocentrismo que implica el asumir sin más que lo que sea que están analizando debe ser ponderado en función de los parámetros del pensamiento burgués. Por el otro, la comparación también constituye "una tarea de contextualización de la información orientada a abordar un interrogante" (Kuper, 2002, p. 161). En efecto, la comparación es el recurso clave para determinar los contextos en que es preciso abordar cada problema (Balbi, 2015, 2017a; Sanjek, 2002; Melhuus, 2002). ${ }^{8}$ En ambos sentidos, el recurso a la comparación está íntimamente relacionado con el holismo y la restricción de la preselección de la información a relevar, ya que las hipótesis que permiten dar sentido a esa información aparentemente inconexa en términos totalizadores suelen ser producto de los ejercicios comparativos. Así, tanto las comparaciones sobre distintos materiales referidos a un mismo medio social — que arrojan luz sobre regularidades, variaciones, conexiones y correlaciones no siempre esperadas-como las que los ponen en relaciones analíticas con fenómenos sociales de otros lugares —que, al introducir nuevos parámetros analíticos, abren nuevos interrogantes y sugieren nuevos puntos de vista- son fuentes decisivas de las preguntas e hipótesis de los etnógrafos, así como de las pequeñas $y$, a veces, grandes correcciones que introducen en la orientación teórica de sus análisis (Balbi, 2015, 2017a).

\section{La teoría puesta en tensión y relativizada}

Considerados en su conjunto, la aspiración de integrar las perspectivas nativas en el análisis, el trío holismo / contextualización / reducción de la preselección de la información al mínimo posible y la comparación hacen de la etnografía una modalidad de investigación potencialmente muy dinámica, en el sentido de que tiende a la revisión de sus orientaciones teóricas y resultados parciales más que a la reafirmación de resultados ya implícitos en aquellas (Shah, 2017). Esto, precisamente, es a lo que se refería Palmeira: la teoría queda sometida a tensión al ser puesta en juego como un factor integral de la construcción de la etnografía en cuanto descripción analítica. Cualesquiera que sean sus orientaciones teóricas específicas, si el etnógrafo procede de esa manera, éstas siempre afrontan algún nivel de riesgo porque se encuentran invertidas en los hechos más que impuestas sobre ellos.

En suma, invertir la teoría en la investigación etnográfica equivale a someterla a una serie de procedimientos de construcción y análisis de datos —distinguibles a fines analíticos pero que en realidad se interpenetran- que le plantean preguntas imprevistas, la hacen súbitamente inapropiada en uno u otro aspecto, la desafían y hasta la increpan. Bien puede decirse que en la etnografía se le

\footnotetext{
${ }^{8}$ Me refiero aquí a contextos que exceden al de origen de cada pieza de información, el cual, como ya he dicho, siempre debe ser tenido en cuenta.
} 
concede, no a lo fenoménicamente 'real' sino a un real construido teóricamente, ya que no la iniciativa de que hablaban Bourdieu y sus colaboradores, al menos una capacidad de respuesta significativa. El trabajo teórico se torna, entonces, inherente a la labor etnográfica.

\section{Dos breves recorridos teóricos por la vía de la etnografía}

La historia de la antropología ofrece innumerables ilustraciones del punto que acabo de establecer. La más adecuada sería, seguramente, la forma en que, en el curso de sus investigaciones etnográficas dedicadas respectivamente a los Nuer y los Tallensi, Edward Evan Evans-Pritchard y Meyer Fortes establecieron hacia 1940 un enfoque centrado en el análisis de los sistemas políticos a través de la reformulación de los recursos analíticos de la teoría estructural-funcionalista de A. R. Radcliffe-Brown, quien daba cuenta del problema del mantenimiento del orden social analizando la estructura y regulación de las relaciones diádicas (fundamentalmente de parentesco), para poner el foco en las relaciones entre grupos que ejercían el control sobre territorios (Fortes y Evans-Pritchard, 1979; Evans-Pritchard, 1977; Fortes, 1945). Otro excelente ejemplo es el desplazamiento de Eric Wolf desde la ecología cultural de Julian Steward hacia una cierta lectura del marxismo a través de una serie de análisis etnográficos desarrollados entre las décadas del sesenta y el setenta del siglo pasado (Wolf, 2001). También sería productivo mostrar cómo Maurice Godelier revisó drásticamente algunas ideas-fuerza del marxismo en el curso de sus investigaciones sobre los Baruya de las Tierras Altas de Nueva Guinea, desarrolladas entre 1967 y fines de la década siguiente, desembocando en una teorización propia acerca de las relaciones entre lo material, lo simbólico y lo imaginario (Godelier, 1986, 1989). Y, aunque no se basa en el trabajo de campo etnográfico sino en una lectura etnográfica de fuentes documentales referidas a la expansión del colonialismo europeo en el Océano Pacífico, cabría mencionar el esfuerzo desarrollado por Marshall Sahlins para introducir una dimensión diacrónica en el estructuralismo de raíz levistraussiana (Sahlins, 1988, 1981).

Sin embargo, consideraciones relativas a la extensión y a las formas habituales en la escritura académica actual me hacen pensar que es preferible presentar dos ejemplos, mucho más modestos, extraídos de mis propias investigaciones. Lo haré sin detenerme realmente en los detalles, tanto etnográficos como teóricos (que el lector podrá encontrar en mis publicaciones previas), con la sola intención de mostrar dos momentos en que la inversión de la teoría en los hechos me condujo a ponerla en tensión y, así, a reformularla.

Hacia 1995 me encontraba abocado a analizar los conflictos que se desarrollaban entre los asociados de una cooperativa de pescadores comerciales de la ciudad de
Victoria, en la provincia de Entre Ríos. Estaba interesado en dar cuenta del patrón que seguían esos conflictos y establecer cómo incidían sobre la posición ocupada por la organización en el proceso productivo pesquero. ${ }^{9} \mathrm{El}$ patrón mencionado involucraba una compleja articulación entre la diferenciación socioeconómica de las unidades productivas de base doméstica de los asociados y un complejo entramado de relaciones personales (parentesco, amistad, relaciones de dependencia personal, etc.) que atravesaban la cooperativa. El hecho que aquí quiero recuperar es que, mientras los asociados disputaban continuamente por el control de la cooperativa (que comportaba la capacidad de establecer sus políticas comerciales y decidir sobre el manejo de sus recursos materiales y monetarios), sistemáticamente expresaban sus opiniones sobre los asuntos de la organización (tanto en ocasiones formalizadas tales como reuniones del Consejo de Administración o las asambleas como en el transcurso de las actividades cotidianas) apelando a una serie de términos extraídos de la doctrina cooperativista: solidaridad, igualdad, democracia, participación, excedentes, ${ }^{10}$ etc. En la práctica, esto implicaba que cursos de acción institucional claramente opuestos que eran seguidos según quiénes controlaran la cooperativa en uno u otro momento fueran presentados en los mismos términos. Por ejemplo, el uso de los excedentes y de subsidios obtenidos por la organización era presentado como igualitario tanto si se los destinaba a financiar la compra de bienes de capital de uso común (un barco de acopio de pescado, un pequeño camión térmico) como si se los usaba para financiar la adquisición de redes y botes por parte de los pescadores cuyas unidades productivas estaban en peores condiciones económicas.

Siguiendo ciertas orientaciones teóricas, durante algún tiempo estuve convencido de que las apelaciones a la jerga del cooperativismo no eran sino una retórica adecuada en ese contexto para justificar cursos de acción que, en último análisis, respondían a los intereses de uno u otro grupo de asociados. Sin embargo, el mandato disciplinario de tomar en serio las perspectivas nativas me llevó a dudar de mi punto de vista, que parecía ser contradicho por numerosas observaciones. Por ejemplo, algunos de los pescadores que, en nombre de la igualdad, impulsaban políticas que los beneficiaban más que a otros asociados, habían permanecido fieles a la cooperativa en momentos en los que claramente les habría convenido vender su producción a los acopiadores que eran sus principales competidores; asimismo, esos hombres estaban entre los más firmes defensores de la práctica, seguida sistemáticamente por la cooperativa, de pagar a los no-socios más de lo que pagaban los acopiadores cada vez que se hacía necesario recurrir a la compra de

\footnotetext{
${ }_{9}$ Sobre los conflictos en la cooperativa véanse: Balbi (2017b, 2000), Boivin, Rosato y Balbi (1999). Sobre el proceso productivo pesquero, véase: Boivin, Rosato y Balbi (2008).

${ }^{10}$ En esta sección empleo las itálicas para denotar las nociones de los actores que protagonizaron los procesos sociales que fueron objeto de mis análisis etnográficos.
} 
pescado para completar una entrega que no era posible cubrir con la producción de los asociados. Observaciones como estas me imponían la reconsideración de mi mirada, que tendía a restarle valor a la adhesión de los asociados a los postulados del cooperativismo. Me faltaba, sin embargo, una inspiración teórica adecuada.

Esa orientación llegaría de la mano de la lectura que hacía el antropólogo argentino Eduardo Archetti de los trabajos dedicados por Émile Durkheim al tema de la moral, la cual enfatizaba su paulatino acercamiento hacia una consideración de ésta en términos que hoy llamaríamos cognitivos. ${ }^{11}$ Esto abría para mí posibilidades analíticas nuevas: acaso lo que ocurría en la cooperativa fuera que los asociados se representaban sus intereses en términos moralmente marcados. Sin embargo, a poco de comenzar a trabajar en esta línea advertí que la mirada de Archetti estaba orientada a priorizar el análisis de elecciones individuales: en efecto, si la moral está directamente relacionada con cómo entendemos el mundo social y ese entendimiento está abierto a interpretaciones, es de esperar que la moral también lo esté y que los individuos se encuentren ante la posibilidad de elegir entre diversos cursos de acción entendidos como moralmente adecuados. Mi problema era que, si bien la existencia de distintas interpretaciones posibles de los postulados morales me interesaba tanto como a Archetti, yo no estaba preocupado por las elecciones individuales sino por las formas en que los asociados se imponían cursos de acción los unos a los otros estableciéndolos como moralmente adecuados.

Esta diferencia crucial me llevó a trabajar por mí mismo en torno de los cambios advertidos por Archetti en la perspectiva de Durkheim, cosa que hice centrándome en el hecho de que, si en sus primeros trabajos el sociólogo francés había definido a la moral en torno a la noción de obligación (haciéndose eco del tema kantiano del imperativo categórico), posteriormente le había sumado la idea de que, para incidir sobre la voluntad de los individuos, los postulados morales deben plantear cursos de acción que les resulten deseables (razonamiento que recupera el tema del bien, propio de la tradición aristotélica). Retomando el par obligación / deseabilidad, mi análisis de los conflictos en la cooperativa pasó así a centrarse en las maneras en que los cursos de acción que convenían a uno $u$ otro grupo de asociados eran planteados primero como deseables en términos de lo que supuestamente sería propio del accionar de una cooperativa para, luego, ser establecidos como obligatorios mediante resoluciones del Consejo de Administración y respaldados por sanciones. Al cabo, llegué a darme cuenta de que la cuestión no era si los asociados adherían o no a los postulados del cooperativismo sino, más bien, cómo se definían e

\footnotetext{
${ }^{11}$ El punto central de su lectura de Durkheim se encuentra en Archetti (1997). He reseñado en detalle mi apropiación de las ideas de ambos autores en Balbi (2016).
}

imponían — de manera más o menos duradera según el caso - interpretaciones socialmente eficaces respecto del sentido de dichos postulados. Dicho de otra manera, los conflictos en torno del control de la cooperativa, sus recursos y sus políticas eran, en parte, conflictos por la definición de los conceptos de democracia, solidaridad, etc., los cuales aparecían como valores morales sostenidos por los asociados a pesar de que sus sentidos no eran necesariamente compartidos por todos ellos y eran siempre inestables. Una vez que llegué a este punto, estuve en condiciones de tomar en serio las perspectivas de los asociados y debo decir - aunque no pueda extenderme en detalles- que recién entonces conseguí aprehender plenamente la dinámica de los conflictos que los enfrentaban y, a la vez, los factores que los inducían a mantenerse agrupados bajo la forma organizativa de la cooperativa.

En este pequeño episodio de mi trayectoria es posible advertir cómo el trabajo etnográfico fue, a la vez, un trabajo de construcción y análisis de datos y un trabajo teórico. En efecto, no sólo mi etnografía dio un salto cualitativo sino que establecí el primer paso de lo que sería mi labor principal durante algo más de veinte años: el desarrollo de una perspectiva antropológica sobre la moral a través de una serie de análisis etnográficos de diversos temas en el terreno de la antropología de la política y de sucesivas revisiones de mis análisis etnográficos previos dedicados a la producción pesquera entrerriana y la cooperativa.

En el siguiente paso de ese recorrido, inicié en 1998 una investigación sobre el lugar ocupado por el concepto de lealtad en las prácticas políticas de los peronistas, abordándolo como un valor moral específicamente suyo y examinando sus orígenes, los procesos sociales que lo impusieron como tal y los que reproducían esa condición, las formas en que orientaba y condicionaba el hacer política de los actores, y finalmente, los 'usos' prácticos a que era sometido (Balbi, 2007). Partiendo de los resultados de mi trabajo anterior, pude comprobar que, debido precisamente a sus fuertes asociaciones morales, el concepto de lealtad, sus opuestos (los de traición y deslealtad) y sus derivados (leal, traidor, etc.) eran recursos clave desplegados por los actores para posicionarse en términos moral y, por ende, políticamente positivos, así como para colocar a sus rivales en posiciones moral y políticamente negativas.

A lo largo de mi investigación me encontré crecientemente sorprendido por la eficacia de esos usos, así como por su muy escasa variedad que, a su vez, revelaba que los sentidos asociados al concepto de lealtad eran sistemáticamente los mismos, como si no hubiera disputas al respecto. Más sorprendente aún me resultaba la constatación de que los usos contemporáneos de esas nociones, así como los sentidos que denotaban, eran casi idénticos a los que revelaban las fuentes primarias y 
secundarias sobre las décadas formativas del peronismo. La lealtad era sistemáticamente considerada como: una virtud de carácter moral propia de todo auténtico peronista; una cualidad inherente a las personas, que se tiene o no se tiene; el fundamento último de las relaciones entre compañeros, la conducción política y la unidad de propósitos de los peronistas; dotada de un carácter asimétrico, en el sentido de que la lealtad del conductor engendraría la de sus seguidores y no al contrario; etc. En todos los casos que mis fuentes exhibían, los usos políticos de la lealtad se ajustaban estrechamente a estos sentidos: los actores denotaban su lealtad (para con sus jefes políticos, agrupaciones, compañeros, el Partido, etc.) mediante una paleta limitada de declaraciones verbales y recursos no verbales (incluyendo complejas puestas en escena fuertemente estandarizadas), y se valían de recursos similares e igualmente limitados para acusar a sus rivales de ser traidores o, más veladamente, desleales. No pude encontrar caso alguno en que las cargas morales de los términos en cuestión fueran invertidas.

El panorama que exhibían mis materiales era, entonces, el de la existencia de un cierto universo conceptual centrado en un valor moral cuyos sentidos no parecían ser disputados y se integraban en un repertorio de usos políticos prácticos o interesados sumamente pobre, todo lo cual parecía no haber experimentado cambios significativos desde mediados de la década de 1940. Esto surgía de mi trabajo en función de dos de las orientaciones analíticas básicas de la etnografía ya mencionadas: la contextualización, que me había impulsado a examinar esos sentidos y usos de los conceptos en diversos contextos sociohistóricos, y el recurso a la comparación, en este caso desplegado en busca de diferencias significativas relacionadas con esos distintos contextos. Sin embargo, esos resultados parciales se me hacían sorprendentes al punto de parecerme imposibles, pues los antropólogos de mi generación estamos más bien preparados para esperar disputas por el sentido, una polisemia generalizada y una creatividad práctica ilimitada que, rutinariamente, se supone asociada a la agencia.

Lo que finalmente intervino en mi auxilio fue la articulación entre la orientación holística de mi investigación y el reconocimiento de que los materiales de que disponía ponían en tela de juicio una parte de mis orientaciones teóricas. En efecto, durante años — siguiendo otro procedimiento establecido en nuestra disciplina- había procurado exégesis nativas del concepto de lealtad y los otros que se le asocian, transitando en vano fuentes documentales y estudios publicados (que abarcaban toda la historia del peronismo), esperando sin suerte que los actores con que trataba las desarrollaran por su cuenta e incluso fracasando reiteradamente en mis intentos de inducirlos a que lo hicieran. Todo lo que había encontrado eran unos pocos pasajes de discursos y de textos firmados por Perón que esbozaban los sentidos del concepto, y su sistemática reiteración, casi siempre textual, en los dichos o escritos de Eva Perón y de innumerables dirigentes y militantes de todos los tiempos. Así las cosas, yo estaba convencido de que las exégesis existían y de que era yo quien no sabía encontrarlas. Sin embargo, la relectura de un texto donde Fredrik Barth (1975) pone en duda el supuesto de que los actores siempre dispongan de exégesis de los símbolos con que operan me condujo a preguntarme si, más que en mis materiales, el problema no radicaría en mis expectativas teóricamente informadas. La decisión de establecer esa posibilidad como una hipótesis de trabajo me condujo a preguntarme cómo aprendían los peronistas lo que significaba la lealtad y a descubrir que lo hacían en la práctica, de maneras tácitas que comprendían a la experiencia de los usos del concepto en el curso de su hacer política y al conocimiento de una serie de 'ejemplares de lealtad', historias estandarizadas que proporcionaban ejemplos paradigmáticos que condensaban los sentidos del concepto (narrativas estereotipadas que se centraban en la relación entre Perón y el pueblo, las relaciones de Eva Perón con su marido y con el pueblo, relatos sobre los militantes de los tiempos de la Resistencia peronista, etc.).

Concluí, entonces, que los peronistas sabían qué era la lealtad -qué implicaba el concepto y, también, cómo usarlo - sin necesidad de contar más que con algunas fórmulas estereotipadas para hablar al respecto. Este estado de cosas hacía que carecieran de estímulos para desarrollar exégesis del concepto $y$, por ende, que no elaboraran los medios necesarios para hacerlo. En consecuencia, sus sentidos variaban muy escasamente y sus usos prácticos, interesados, se ajustaban a unos pocos procedimientos que, al ser sumamente eficaces, desalentaban ulteriores resignificaciones (consideraciones que cabe extender a los demás términos mencionados: traición, deslealtad, etc.). En suma, una vez que fui capaz de revisar algunas de mis orientaciones teóricas en función de mis materiales, pude - con bastante demoradesarrollar una mirada totalizadora en la que mi análisis sobre cuestiones que inicialmente no había considerado como las del aprendizaje y la escasa elaboración discursiva de los sentidos del concepto de lealtad arrojaba luz sobre aquellos otros rasgos aparentemente sorprendentes de mis materiales que eran la ausencia de variaciones en sus sentidos y usos predominantes a lo largo de más de cuarenta años.

Cabe agregar que este episodio me condujo a reorientar mi perspectiva teórica sobre el análisis antropológico de la moral hacia el problema de las relaciones entre su aprendizaje y las distintas formas en que ella se vincula con el comportamiento. Esto, a su vez, me permitió volver sobre mis materiales referidos a la cooperativa de pescadores (Balbi, 2016) para revisar mi análisis previo y dar cuenta de la estabilidad y eficacia relativamente mayores de los sentidos asociados a los conceptos de cooperativa y excedentes - vinculados con la experiencia prolongada de la explotación de los pescadores por 
los acopiadores y la consecuente valorización del cooperativismo como recurso para combatirla-, que contrastaba con los sentidos cambiantes de los de democracia, igualdad, etc.

\section{La perspectiva etnográfica}

Lo dicho hasta este punto impone la pregunta sobre qué es, en última instancia, lo que predispone o induce a los antropólogos a poner en tensión y relativizar la teoría en el curso de sus etnografías. Se trata de un interrogante cuyo tratamiento desborda ampliamente a estas páginas, por lo que me limitaré a esbozar una respuesta que será necesario desarrollar in extenso en otra oportunidad.

Un buen punto de partida puede ser la siguiente observación de Laura Nader (2011):

"La etnografía, sea lo que sea, nunca ha sido mera descripción. Es también teórica en su modo de descripción. En verdad, la etnografía es una teoría de la descripción." (p. 211; los énfasis son del original)

Ciertamente, con las salvedades que son de rigor, puede decirse que la etnografía comporta tres apuestas analíticas interrelacionadas. Primero, la apuesta en favor de la centralidad analítica de la variabilidad de los hechos sociales (lo que suele ser llamado 'diversidad' o 'diferencia'), que se refleja en la atención brindada a sus detalles y a sus interconexiones con otros hechos producidos en su contexto de origen. Segundo, la apuesta por el valor estratégico del análisis de las perspectivas nativas para dar cuenta de los hechos sociales, que se traduce en el intento de integrarlas en la etnografía según ya hemos visto. Y, finalmente, la apuesta en favor de la productividad de abordar los hechos sociales sin segmentarlos en base a criterios a priori, que conduce a reducir al mínimo posible la preselección de la información a relevar a fin de poder detectar y analizar conexiones inesperadas entre distintos fenómenos. ${ }^{12}$ Combinadas, estas tres apuestas conforman, en efecto, lo que Nader llama una teoría de la descripción.

Lo que falta en esta fórmula es advertir que esa teoría de la descripción es el correlato metodológico de un teoría muy general sobre la naturaleza de la vida social desarrollada a lo largo de la historia de la disciplina (Holy, 1984). Me refiero a lo que bien cabe denominar la perspectiva etnográfica, una concepción teórica general, que se presenta en versiones muy diversas, de la vida social como necesariamente variable (tanto entre distintos medios sociales y culturales como en cada uno de ellos), continua (en el sentido de no existen soluciones de continuidad empíricas entre los fenómenos sociales) y protagonizada activamente por sujetos socialmente

\footnotetext{
12 Sobre la diversidad y el holismo en cuanto construcciones teóricas, véase: Guber (1991)
}

situados (esto es, no por seres humanos abstractos) cuya racionalidad específica debe ser materia de análisis empírico. ${ }^{13}$ Esta perspectiva, que nació al abrigo de la antropología clásica, ha sido reproducida (en el doble sentido de mantenida y reformulada) hasta nuestros días por una serie de factores que no puedo examinar aquí en detalle pero entre los cuales destacan su sostenida productividad (es decir, su valor heurístico intrínseco) y su importancia para el mantenimiento de los límites de la disciplina. Independientemente de la opinión de cada antropólogo respecto del uso de teoría, toda investigación etnográfica es teórica en el sentido de que se funda en esta teoría mínima de lo social que es la perspectiva etnográfica. ${ }^{14}$

A despecho de todos los puntos de controversia que nos dividen en torno de esas cuestiones (tantos que no puedo siquiera esbozarlos aquí), los antropólogos tendemos a coincidir, en el nivel más básico, en que los fenómenos sociales se caracterizan por su variabilidad, se relacionan unos con otros sin que existan soluciones de continuidad y son protagonizados por sujetos socialmente situados que son portadores de distintas racionalidades. Y solemos coincidir, sobre todo, en que todo esto es materia de investigación empírica y no puede ser abordado mediante la adopción de primeros principios y el despliegue de modelos deductivos ni a través de la adopción de teorías que supuestamente permitirían determinar a priori cuál sería la información relevante, cómo pueden o no pueden ser los hechos sociales y cómo tendríamos que abordarlos.

Este último punto de coincidencia supone ya la puesta en acción de esa teoría muy básica sobre la naturaleza de la vida social que es la perspectiva etnográfica, su deriva hacia el plano de la metodología, es decir, de la aproximación teóricamente informada y articulada a la producción de datos (Ellen, 1984): en efecto, si las tres apuestas analíticas ya referidas conforman la teoría de la descripción de que hablaba Nader, ello sucede porque traducen la perspectiva etnográfica en términos metodológicos. En suma, la perspectiva etnográfica proporciona las orientaciones de procedimiento básicas que estructuran la totalidad de la labor etnográfica ${ }^{15}$

\footnotetext{
${ }^{13}$ Kirsten Hastrup (1997) afirma el carácter teórico de la antropología desde un punto de vista que difiere del aquí planteado aunque no es incompatible con éste. Asimismo, en otro trabajo, Hastrup (2005) ofrece una versión de lo que yo llamo 'perspectiva etnográfica' que articula la dimensión teórica con la experiencial: "En la antropología, la 'etnografía' (...) no es simplemente un método (un sinónimo para trabajo de campo) ni una descripción particularmente densa de realidades locales. Ambas cosas son subsumidas por una sensibilidad particular para con el mundo, un modo de percepción que incluye, por un lado, una conciencia reflexiva de y un respeto por las particularidades y complejidades locales, y por el otro, la intervención teorética implicada por la representación" (p. 141)

${ }^{14}$ Entiendo que los colegas que se esfuerzan por trabajar sin teoría, así como los que piensan que hacen un uso meramente instrumental de ella (esto es, eligiendo a posteriori herramientas teóricas adecuadas para abordar cuestiones que, según ellos, surgen 'del campo') fallan en reconocer la naturaleza teórica de las orientaciones más básicas que rigen la totalidad de su trabajo. Véase: Balbi (2012, 2015, 2017a). ${ }^{15}$ Un corolario de esto es la imposibilidad de la existencia de lo que se
} 
y dan lugar a esa inversión de la teoría en los hechos de que hablaba Palmeira. Así, cabe concluir que la perspectiva etnográfica y la inversión de la teoría en los hechos corresponden a sendos niveles jerárquicamente interrelacionados de la conformación de la etnografía en cuanto una empresa cuya naturaleza es eminentemente teórica.

Buenos Aires, 24 de septiembre de 2019

\section{Agradecimientos}

He presentado una primera aproximación al tema de este artículo en mi intervención en el Panel "Entre la determinación y la iluminación ¿Cómo funciona la teoría con el material de campo?", desarrollado el 11 de agosto de 2016 en el marco de las VIII Jornadas sobre etnografía y Métodos Cualitativos organizadas, por el Centro de Antropología Social - Instituto de Desarrollo Económico y Social. Agradezco especialmente a Ana Rosato - que, a pesar de que han transcurrido más de treinta años, sigue enseñándome antropología- por haberme sugerido la lectura del texto de Moacir Palmeira. Quisiera expresar también mi agradecimiento a los dos colegas que evaluaron en forma anónima la primera versión de este artículo.

\section{Bibliografía}

Archetti, E. (1997). The moralities of argentinian football. En: Howell, S. (ed.). The ethnography of moralities (pp. 99-125). London and New York: Routledge.

Balbi, F. A. (2017a): Servidumbre y emancipación de la comparación. En Balbi, F. A. (Ed.), La comparación en Antropología Social: problemas y perspectivas (pp. 2976). Buenos Aires: Editorial Antropofagia.

Balbi, F. A. (2017b). Comparación, etnografía y generalización. Anuário Antropológico (42)1: 9-35. doi: 10.4000/aa.1628

Balbi, F. A. (2017c). Moral e interés. Una perspectiva antropológica. PUBLICAR en Antropología y Ciencias Sociales, Año XIV, No. XXIII: 9-30. Recuperado de http://ppct.caicyt.gov.ar/index.php/publicar/article/ view/11710/45454575757808

ha dado en llamar 'teoría etnográfica' (Da Col y Graeber, 2011). Esta idea es epistemológicamente ingenua puesto que, como ya se dijo, las teorías no proceden de los hechos sino que su propio despliegue es lo que da lugar a la producción teórica a través de un camino que -al menos en la etnografía - está jalonado de hechos. De lo que se trata en etnografía es, entonces, de apelar a orientaciones teóricas que nos permitan pensar de una manera etnográficamente sensible (Balbi, 2015), es decir, atenta a la naturaleza de la vida social como variable, continua y protagonizada activamente por sujetos socialmente situados. Este es el primer prerrequisito para la producción de teoría en el curso de la etnografía - que no es lo mismo que la producción de teoría supuestamente 'etnográfica'-.
Balbi, F. A. (2016). A moral como parte integral da produção da vida social. Uma leitura heterodoxa dos escritos de Emile Durkheim. Sociologia \& Antropologia, 6(3): 755-778. doi: http://dx.doi.org/10.1590/2238$38752016 \mathrm{v} 638$

Balbi, F. A. (2015). Retratistas de mariposas. Acerca del lugar subordinado de la comparación en la antropología social y cultural contemporánea. Revista del Museo de Antropología, 8(1): 171-186. doi: http://dx.doi. org/10.31048/1852.4826.v8.n1.9516

Balbi, F. A. (2012). La integración dinámica de las 'perspectivas nativas' en la investigación etnográfica. Intersecciones en Antropología, 14: 485-499. Recuperado de http://www.redalyc.org/articulo.oa?id=179525429013

Balbi, F. A. (2007). De leales, desleales y traidores. Valor moral y concepción de política en el peronismo. Buenos Aires: Editorial Antropofagia.

Balbi, F. A. (2000). Interdependencia, memoria institucional y valores morales: fundamentos sociales de la moralidad en una cooperativa de pescadores entrerrianos. Avá. Revista de Antropología Social, 2: 95-111.

Bachelard, G. (1979). El racionalismo aplicado. Buenos Aires: Paidós.

Barth, F. (1975). Ritual and knowledge among the Baktaman of New Guinea. Oslo-New Haven: Universitetsforlaget-Yale University Press.

Bloch, M. (2005). Where did anthropology go? Or the need for 'human nature'. Essays on cultural transmission, (1-19). Oxford: Berg.

Boivin, M., Rosato, A. y Balbi, F. A. (eds.). 2008: Calando la vida. Ambiente y pesca artesanal en el Delta entrerriano. Buenos Aires: Editorial Antropofagia.

Boivin, M., Rosato, A. y Balbi, F. A. (1999). Conflictos políticos y valores morales: usos simbólicos del modelo cooperativista. Etnía, 43: 171-188.

Bourdieu, P., Chamboredon, J. y Passeron, J. (1986). El oficio de sociólogo. México: Siglo XXI.

Cook, J. (11 de mayo de 2016). Ethnography: Translation [Correspondences, Cultural Anthropology website]. Recuperado de https://culanth.org/fieldsights/874ethnography-translation

Da Col, G. (2017). Two or three things I know about ethnographic theory. Hau: Journal of Ethnographic Theory, 7(1): 1-8. doi: 10.14318/hau7.1.002

Da Col, G. y Graeber, D. (2011). Foreword: The return 
of ethnographic theory. Hau: Journal of Ethnographic Theory, 1(1): vi-xxxv. doi: 10.14318/hau1.1.001

Das, V., Jackson, M., Kleinman, A. y Singh, B. (2014). Introduction. Experiments between Anthropology and Philosophy: Affinities and antagonisms. En Das, V., Jackson, M., Kleinman, A. y Singh, B. (Eds.), The Ground Between. Anthropologists engage philosophy (pp. 1-26). Durham and London: Duke University Press.

Ellen, R. (2010). Theories in anthropology and 'anthropological theory'. Journal of the Royal Anthropological Institute (N.S.), 16(2): 387-404. Recuperado de http://www.jstor.org/stable/40606106

Ellen, R. (1984). Introduction. En Ellen, R. F. (Ed.), Ethnographic research. A guide to general conduct (pp. 1-12). London: Academic Press.

Escobar, A. y Restrepo, E. (2005). "Otras antropologías y antropologías de otro modo": elementos para una red de antropologías del mundo. En: Escobar, E., Más allá del Tercer Mundo. Globalización y Diferencia (pp. 231-256). Bogotá: Instituto Colombiano de Antropología e Historia.

Evans-Pritchard, E. E. (1977). Los Nuer. Barcelona: Anagrama.

Fox, R. G. y Gingrich, A. (2002). Introduction. En Gingrich, A. y Fox, R. G. (Eds.), Anthropology, by comparison (pp. 1-24). London and New York: Routledge.

Fortes, M. (1945). The dynamics of clanship among the Tallensi. London: International African Institute - Oxford University Press.

Fortes, M. y Evans-Pritchard, E. E. (1979). Sistemas políticos africanos. En Llobera, J. (comp.), Antropología política (pp. 85-105) Barcelona: Anagrama.

Godelier, M. (1986). La producción de Grandes Hombres. Poder y dominación masculina entre los Baruya de Nueva Guinea. Marid: Akal.

Godelier, M. (1989). Lo ideal y lo material. Pensamiento, economías, sociedades. Madrid: Taurus.

Guber, R. (2016). Conocimiento antropológico, trabajo de campo y patetismo editorial. Cuestión de escala y de tiempo. [En sus propios términos. World Council of Anthropological associations website]. Recuperado de https://www.wcaanet.org/publications/propios_terminos/ guber.shtml

Guber, R. (1991). El salvaje metropolitano. Buenos Aires: Legasa.

Gupta, A. y Ferguson, J. (1997). Discipline and practice:
"The field" as site, method, and location in Anthropology. En: Gupta, A. y Ferguson, J. (Eds.), Anthropological locations: boundaries and grounds of a field science (pp. 1-46). Berkeley, Los Angeles and London: University of California Press.

Hastrup, K. (2005). Social anthropology. Towards a pragmatic enlightenment? Social Anthropology, 13(2), 133-149. doi: 10.1017/S0964028205001199

Hastrup, K. (1997). The dynamics of anthropological theory. Cultural Dynamics, 9(3), 351-371. doi: 10.1177/092137409700900305

Holy, L. (1984). Theory, methodology and the research process. En Ellen, R. F. (Ed.), Ethnographic research. A guide to general conduct (pp. 13-35). London: Academic Press.

Howell, S. (2018). Ethnography. En The Cambridge Encyclopedia of Anthropology. Recuperado de http:// doi.org/10.29164/18ethno

Howell, S. (2017). Two or three things I love about ethnography. HAU: Journal of Ethnographic Theory, 7(1): 15-20. doi: 10.14318/hau7.1.004

Ingold, T. (2017). ¡Suficiente con la etnografía! Revista Colombiana de Antropología, 53(2): 143-159. Recuperado de http://www.redalyc.org/articulo.oa?id=105052402007

Ingold, T. (2008). Anthropology is not ethnography. Proceedings of the British Academy, 154: 69-92. doi: $10.5871 / \mathrm{bacad} / 9780197264355.001 .0001$

Kuper, A. (2002). Comparison and contextualization: reflections on South Africa. En Gingrich, A. y Fox, R. G. (Eds.), op. cit. (pp. 143-166).

Marcus, G. (25 de mayo de 2016). Ethnography: Integration. [Correspondences, Cultural Anthropology website]. Recuperado de https://culanth.org/ fieldsights/879-ethnography-integration

MacDougall, S. (11 de mayo de 2016). Let's keep ethnography, and talk more about how it's done. [Correspondences, Cultural Anthropology website]. Recuperado de https://culanth.org/fieldsights/877ethnography-deviation

Melhuus, M. (2002). Issues of relevance: anthropology and the challenges of cross-cultural comparison. En Gingrich, A. y Fox, R. G. (Eds.), op. cit. (pp. 70-91).

Miller, D. (2017). Anthropology is the discipline but the goal is ethnography. HAU: Journal of Ethnographic Theory, 7(1): 27-31. doi: 10.14318/hau7.1.006 
Nader, L. (2011). Ethnography as theory. HAU: Journal of Ethnographic Theory, 1(1): 211-219. doi: 10.14318/ hau1.1.008

Palmeira, M. (2011). Prefacio. En: Leite Lopes, J. S. El vapor del diablo. El trabajo de los obreros del azúcar (pp. 57-62). Buenos Aires: Ed. Antropofagia.

Peirano, M. (1992). A favor da etnografía. Série Antropologia, PPAS-UNB, Brasilia. Recuperado de http://www.dan.unb.br/multisites/dan/media/docs/ Serie130empdf

Pitt-Rivers, J. (1973): El análisis del contexto y el "locus" del modelo. En Tres ensayos de antropología estructural (pp. 13-48). Barcelona: Anagrama.

Putnam, H. (1981). Reason, truth and history. Cambridge: Cambridge University Press.

Restrepo, E. (2016). Etnografía: alcances, técnicas y éticas. Bogotá: Envión Editores.

Sahlins, M. (1988). Islas de historia. Barcelona: Gedisa.

Sahlins, M. (1981). Historical Metaphors and Mythical Realities. Structure in the early history of the Sandwich Islands Kingdom. Ann Arbor: The University of Chicago Press.

Sanjek, R. (2002). Ethnography. En Barnard, A. y J. Spencer (Eds.): Encyclopaedia of Social-Cultural Anthropology (pp. 295-302). London and New York: Routledge.

Shah, A. (2017). Ethnography?: Participant observation, a potentially revolutionary praxis. HAU: Journal of Ethnographic Theory, 7(1): 45-59. doi: doi.org/10.14318/ hau7.1.008

Shryock, A. (3 de mayo de 2016). Ethnography: Provocation. [Correspondences, Cultural Anthropology website]. Recuperado de https://culanth.org/ fieldsights/871-ethnography-provocation

Strathern, M. (2004). Partial connections: updated edition. Walnut Creek, CA: AltaMira Press.

Visacovsky, S. (2017). Etnografía y antropología en Argentina: propuestas para la reconstrucción de un programa de investigación de lo universal. Antípoda. Revista de Antropología y Arqueología, 27: 65-91. doi: 10.7440/antipoda27.2017.03

Wolf, E. (2001). On Fieldwork and Theory. En: Pathways of power. Building an anthropology of the modern world (pp. 49-62). Berkeley, Los Angeles, London: The University of California Press.

Yengoyan, A. (2006). Comparison and its discontents. En Yengoyan, A. (Ed.), Modes of comparison: theory and practice (pp. 137-158). Ann Arbor: The University of Michigan Press. 